\title{
Effect of Laser Heating on Nonlinear Surface Deformation of Acoustically Levitated Droplet
}

\author{
Masanori Kawakami $^{2(a)}$, Yutaka Abe ${ }^{1(\mathrm{~b})}$, Akiko Kaneko ${ }^{1(\mathrm{c})}$ and Koji Hasegawa ${ }^{2(\mathrm{~d})}$ \\ University of Tsukuba, ${ }^{1}$ Institute of engineering Mechanics and System, ${ }^{2}$ Graduate \\ school of System and Information Engineering, \\ 1-1-1 Tennoudai, Tsukuba, Ibaraki, 305-8573, Japan \\ mkawakami@edu.esys.tsukuba.ac.jp ${ }^{(a)}$ \\ abe@kz.tsukuba.ac.jp ${ }^{(\mathrm{b})}$ \\ kaneko@kz.tsukuba.ac.jp ${ }^{(\mathrm{c})}$ \\ khasegawa@edu.esys.tsukuba.ac.jp ${ }^{(\mathrm{d})}$
}

\section{Abstract}

Under the microgravity environment, new and high quality materials with a homogeneous crystal structure are expected to be manufactured by undercooling solidification, since the material manufacturing under the microgravity environment has no effect of gravity. However, the temperature change on the interface of the material in space is expected to affect on the material processing due to the changing of physical property corresponding temperature. The purpose of the present study is to investigate effect of the laser heating on surface deformation of large levitated droplet. A water droplet levitated in the acoustic standing wave is heated by YAG laser. In order to increase the water droplet temperature, rhodamine $6 \mathrm{G}$ is solved in it to achieve high absorbance of the laser. Droplet from $2.5 \mathrm{~mm}$ to $5.5 \mathrm{~mm}$ in diameter were levitated and heated. The deformation of the droplet interface has been observed by high speed video camera. We used the radiation thermometer for the measurement of the temperature of droplet surface. It is noticed that the heated droplet deforms with its resonance frequencies. The experimental result of shape oscillation makes it possible to simulate the oscillation.

\section{Introduction}

Containerless technology has been well recognized as a method for obtaining liquid undercooling. Particularly in microgravity condition, liquid can remain undisturbed condition and deep undercooling is achieved. One of the major recent advances for experiments in containerless processing is acoustic levitation by ultrasound. For carrying out containerless processing under the microgravity condition, the larger droplet is more desirable. However the larger droplet levitates, the more deformation occurs and influences the solidification process remarkably.

There are many previous studies relating to droplet levitation. Many researches on oscillations and rotations of a levitated droplet have been done to investigate the interfacial behavior and to measure the physical properties. Marston, P. L. $(1980,1981)$ analyzed the vibration and the static deformation of levitated droplets and bubbles in the fluid with acoustic radiation pressure. Trinh, E. H. et al. (1982) conducted the experiment of the acoustically levitated silicone oil in the water. They identify the resonance frequency for each mode of the oscillation 
of silicone oil. Wang, T. G. et al. (1996) observed the deformation behavior of the rotating levitated droplet in the microgravity environment. Ermoline, A. et al. (2004) heated the ceramic sample of $1-3 \mathrm{~mm}$ in diameter by the laser. The sample sphere is heated up to $3000 \mathrm{~K}$. Position of a heated sample vertically oscillated under the microgravity condition. Xie, W. J. et al. (2002) conducted the $\mathrm{Pb}-\mathrm{Sn}$ solidification by using acoustic levitation. The solidified $\mathrm{Pb}-\mathrm{Sn}$ sample has the ripples on its surface. The crystal structure near the ripples looks like the spreading ripples on a disturbed water surface. It suggests that the oscillation affect the crystal structure.

Although there are many researches on the shape oscillation of acoustically levitated droplet, the effect of the temperature change on the interfacial behavior of droplet is not sufficiently investigated. The purpose of the present study is to investigate the effect of laser heating on nonlinear surface deformation of acoustically levitated droplet from 2.5 to $5.5 \mathrm{~mm}$ in diameter. The deformation and oscillation of heated droplet was observed by high speed video camera.

\section{Experimental Apparatus}

\section{and Procedure}

Figure 1 (a) shows a schematic diagram of the experimental apparatus. The test section is composed of the horn as a solid speaker and the glass plate as a reflector. A sinusoidal wave signal from the function generator is applied to the ultrasonic transducer to emit the ultrasonic wave into the test section. Ultrasonic wave is reflected at the upper glass plate, and standing wave is formed in the test section. The resonance frequency of the transducer is $19.2 \mathrm{kHz}$. The distance between the horn and the reflector is $47.5 \mathrm{~mm}$. The sound pressure level at antinode was set at 160-165 dB. The sound pressure amplitude was

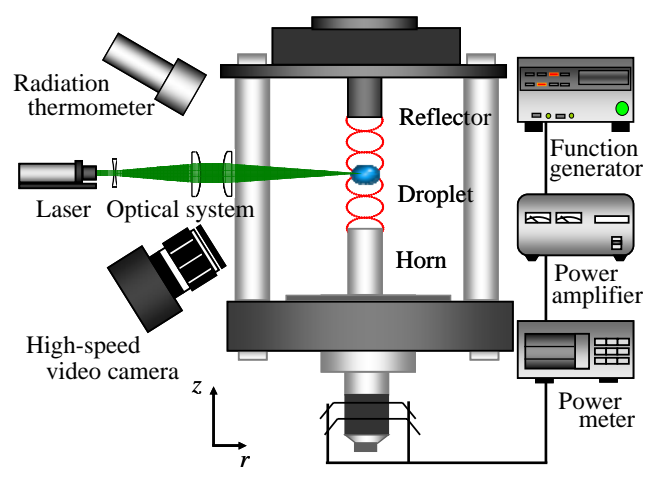

(a) Ultrasonic levitator and measurement equipment

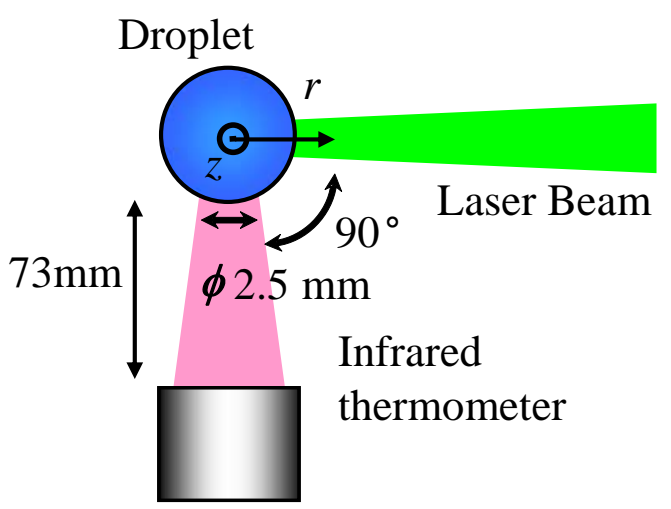

(b) Temperature measurement of a droplet surface

Fig. 1: Schematic diagram of the experimental apparatus

Table 1: Physical properties of water

\begin{tabular}{cccc}
\hline Fluids & $\begin{array}{c}\text { Density } \\
{\left[\mathrm{kg} / \mathrm{m}^{3}\right]}\end{array}$ & $\begin{array}{c}\text { Viscosity } \\
{[\mathrm{mPa} \cdot \mathrm{s}]}\end{array}$ & $\begin{array}{c}\text { Surface } \\
\text { tension } \\
{[\mathrm{mN} / \mathrm{m}]}\end{array}$ \\
\hline $\begin{array}{c}\text { Water } \\
20\left[{ }^{\circ} \mathrm{C}\right]\end{array}$ & 998 & 0.890 & 72.8 \\
$\begin{array}{c}\text { Water } \\
35\left[{ }^{\circ} \mathrm{C}\right] \\
\text { Water } \\
80\left[{ }^{\circ} \mathrm{C}\right]\end{array}$ & 994 & 0.725 & 70.4 \\
\hline
\end{tabular}

measured by the probe microphone (Type 4182 produced by Brüel \& Kjær). Acoustic pressure level in units of decibels $\mathrm{dB}$, a logarithmic relative measure with a defined reference pressure $P_{\text {ref }}=20 \mu \mathrm{Pa}$. The sound pressure level is thus:

$$
S P L=20 \log _{10} P / P_{\text {ref }},
$$


where $P$ was the measured sound pressure. A water droplet is injected at a pressure node by a syringe. The droplet is levitated due to balance between the acoustic radiation force and the gravitational force.

The droplet is heated by YAG laser (Excel Laser produced by Laser QUANTUM Ltd.) with wave length of $532 \mathrm{~nm}$. The maximum power of the laser is $1.58 \mathrm{~W}$. In order to achieve high absorbance of the laser beam in the droplet, $150 \mathrm{mg} / \mathrm{L}$ of rhodamine 6G was solved in the water. Physical properties such as density, viscosity, and surface tension of the droplet are assumed the same as those of water as shown in table 1. When the droplet absorbs the laser light, it emits fluorescence light with a wave length of $550 \mathrm{~nm}$. The interfacial behavior of levitated droplet is recorded by high speed video camera (FASTCAMMax produced by Photron Ltd.). In order to obtain meaningful experimental data, the record speed was set at $1000 \mathrm{fps}$. The scattering light of the laser was cut by an optical filter. The surface temperature of the droplet has been measured by the infrared thermometer (TEMPERATURE HiTESTER 3445 produced by HIOKI E. E. CORPORATION). The measurement spot of the thermometer is $2.5 \mathrm{~mm}$. The spot is perpendicular to the laser beam on the droplet as shown in Fig.1 (b).

\section{Results and}

\section{Discussions}

\section{Effect of Laser Heating on a}

Droplet's Interface and Surface

Temperature

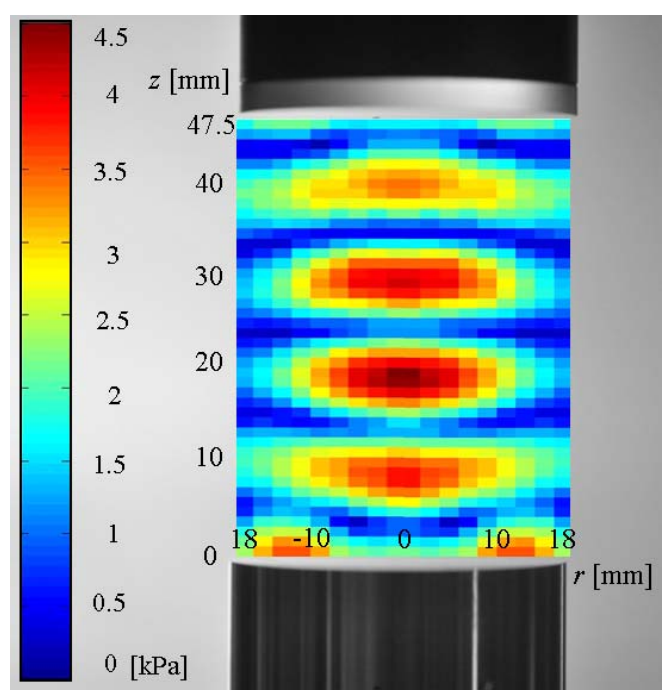

Fig. 2: Sound pressure distribution in the test section

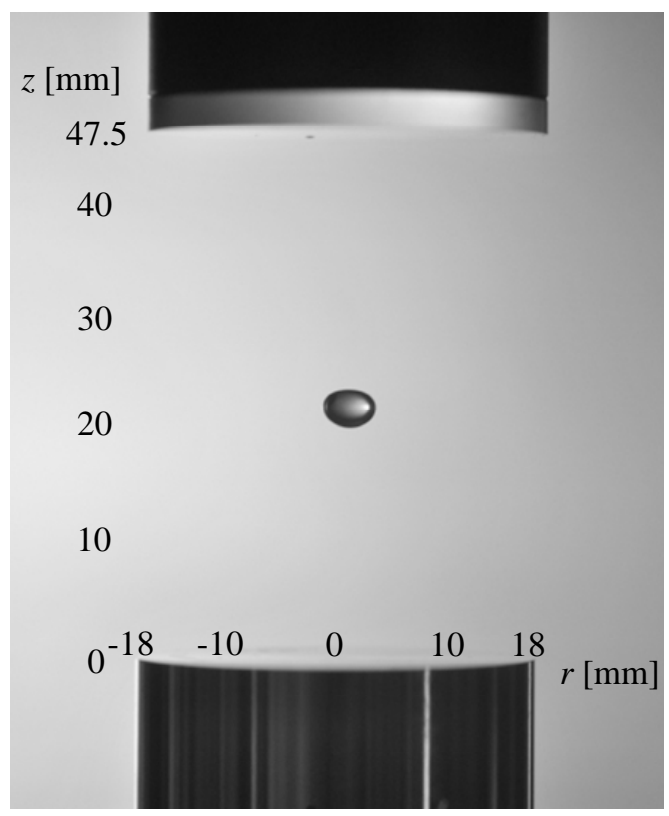

Fig. 3: Levitated water droplet

Figure 2 shows the sound pressure distribution measured by the probe microphone when the sound pressure level at antinode was set at $162 \mathrm{~dB}$. The stably levitated droplet is shown in Fig. 3. In this case, a droplet of $4.3 \mathrm{~mm}$ in diameter was levitated at $162.1 \mathrm{~dB}$. The droplet is levitated in the pressure antinode where the $z$ is about $23 \mathrm{~mm}$. The levitation range of non-heated droplet was experimentally investigated by Kawakami et al. (2009). According to the paper, the stability of a droplet is dominated by the sound pressure and droplet diameter. 
The laser heating was carried out to the stably levitated droplet as shown in Fig. 3. Figure 4 shows the time variation of aspect ratio and temperature of levitated water droplet. The droplet of 5.2 $\mathrm{mm}$ in diameter levitated at $162.1 \mathrm{~dB}$. The droplet is heated by the laser. The estimated irradiated energy was about $0.76 \mathrm{~W}$. The $t$ means time. The open plots and the $T_{\mathrm{s}}$ indicate the surface temperature of water droplet. The temperature rapidly increased right after heating process started. The $T_{\mathrm{s}}$ rose by about $10{ }^{\circ} \mathrm{C}$ in 1 sec. Then, the temperature reached $35{ }^{\circ} \mathrm{C}$ at $22 \mathrm{sec}$. After $22 \mathrm{sec}$, the $T_{\mathrm{s}}$ suddenly decreased by $2{ }^{\circ} \mathrm{C}$ and stayed constant at about $32{ }^{\circ} \mathrm{C}$. There are two reasons for this thermal profile: The first is that the measurement spot of the thermometer was not well-adjusted in the center of the droplet, which is considered that the large deformation and the evaporation underrepresented the temperature. The second reason is that the periodical deformation accelerates heat transfer between droplet and ambient gas.

The solid line indicates the aspect ratio of the droplet. The aspect ratio $\alpha$ is defined as following:

$$
\alpha=\frac{a}{b}
$$

where $a$ is the width and $b$ is the height of a droplet. There was slight increase of the $\alpha$ from 0 sec to $14 \mathrm{sec}$. The droplet becomes flattened. It is considered that the surface tension of water decreased due to temperature rising of the droplet. A heated droplet is difficult to maintain the spherical shape against the sound pressure because the surface tension becomes lower. The fluctuation started after $14 \mathrm{sec}$. The amplitude of deformation became larger from 14 to $20 \mathrm{sec}$. After $20 \mathrm{sec}$, the large fluctuation continued for over 20 sec. Average of maximum value of the $\alpha$ was 4.6 and minimum was 1.0. The typical snapshots of the deformation are shown in Fig. 5. The images are 1 cycle of oscillation. This figure corresponds to the Fig. 4. The periodical large deformation was observed. Few reasons for trigger mechanism of deformation are considered as follows: Surface tension of water decrease due to heating. The inhomogeneous heating generates temperature gradient. And the heated droplet changed the ambient temperature.

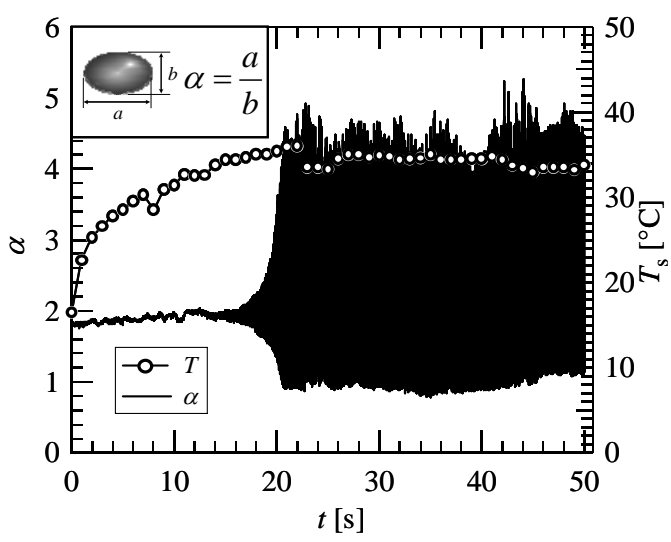

Fig. 4: The time variation of aspect ratio and temperature of levitated water droplet (equivalent diameter: $5.2 \mathrm{~mm}, S P L: 162.1 \mathrm{~dB}$ )

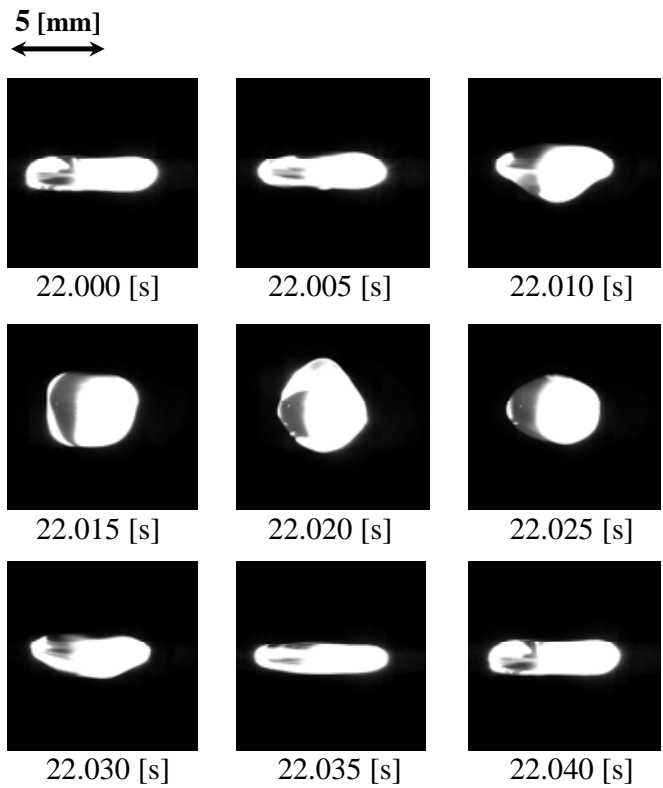

Fig. 5: Typical snapshots of shape oscillation of a levitated droplet

In order to investigate the trend of oscillation occurrence, we conducted a number of experiments such as Fig. 4 and plotted whether shape oscillation started or not. Figure 6 shows correlation between the sound pressure and the equivalent droplet diameter of the heated droplet. The surface temperature of 
the droplet was kept at around $35{ }^{\circ} \mathrm{C}$ by adjusting the power of the laser. Each open plot shows the stably-levitated droplet before and after heating. We use the term "stably" to express that fluctuation of $\alpha$ is within $3 \%$. The solid plots indicate that a shape deformation occurred after heating as shown in Fig. 5.

Danilov, S. D. et al. (1992) proposed the upper limit of the sound pressure $P_{\mathrm{M}}$ to keep the droplet stable as following:

$P_{\mathrm{M}}=\frac{\sqrt{6.8 \sigma_{\mathrm{s}} \rho c^{2}}}{d}$,

where, $d$ is the droplet equivalent diameter, $\sigma_{\mathrm{s}}$ is the surface tension of the droplet, $\rho$ is the density of ambient fluid and $c$ is the sound speed of ambient. The lower limit $P_{\mathrm{m}}$ to sustain a droplet is described by King, L. V. (1934). $P_{\mathrm{m}}$ is as following:

$P_{\mathrm{m}}=\frac{\sqrt{1.6 \rho_{\mathrm{s}} \rho g c^{2}}}{k}$

Here, $k=2 \pi / \lambda$ is the wave number, $\lambda$ is the wavelength, $\rho_{\mathrm{s}}$ is the density of levitated sample and $g$ is the acceleration of gravity. The solid and dashed line in Fig. 6 is derived by equation (3) and (4). The area between solid and dashed line is considered as stably levitated condition. Deformation of the heated droplet tends to occur near the solid line which is the upper limit of levitation. The closed plots were near the solid line. The line is mainly dominated by the surface tension of water droplet as shown in equation (3). When a droplet is heated, the surface tension decreases and the solid line descends. However the displacement of the line is very small due to small variation of $\sigma_{\mathrm{s}}$. The laser beam was irradiated from the one side toward a droplet. It is possible that there is thermal gradient in a droplet. It suggests that the not only the increase of surface tension due to temperature rising, but also laser heating makes it easy to oscillate even in the stable area. Further precise investigation to the trigger mechanism of the oscillation is needed.

Rayleigh, L. (1879) derived the norder mode of resonance frequencies to the following equation:

$$
f_{n}=\frac{1}{2 \pi} \sqrt{n(n-1)(n+2) \frac{\sigma_{\mathrm{s}}}{\rho_{\mathrm{s}} r^{3}}},
$$

where $\sigma_{\mathrm{s}}$ is the surface tension, $\rho_{\mathrm{s}}$ is the density and $r$ is the radius of the droplet. We applied the parameters of $r=2.6 \times 10^{-3}$ $\mathrm{m}, \sigma_{\mathrm{s}}=0.0711 \mathrm{~N} / \mathrm{m}$ and $\rho_{\mathrm{s}}=996 \mathrm{~kg} / \mathrm{m}^{3}$ to the equation (5), which are experimental condition of Fig. 4. The estimated resonance frequencies are $f_{1}=28.7, f_{2}=$ 55.6, $f_{3}=86.1, f_{5}=120.1$ and $f_{6}=157.2$ Hz. Figure 7 shows the FFT analysis of the aspect ratio $\alpha . P\left(f_{n}\right)$ is the power spectrum density of variation of $\alpha$. We analyzed the $\alpha$ in the range from $t=$ $22.000 \mathrm{sec}$ to $26.096 \mathrm{sec}$ in Fig. 4. There are apparent peaks from 2 to 6 order modes. The most prominent point is the 2order mode, which indicates that the $f_{2}$ is $27.1 \mathrm{~Hz}$ and $P\left(f_{2}\right)$ is over $10^{3}$.

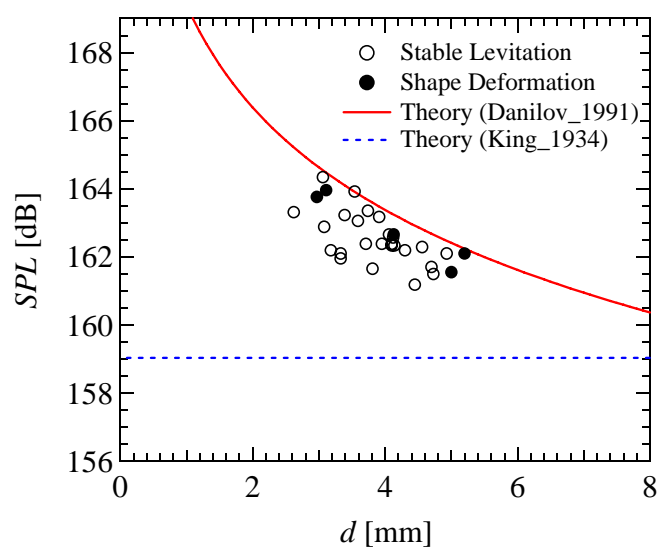

Fig. 6: Correlation between the sound pressure and the equivalent droplet diameter of heated droplet 


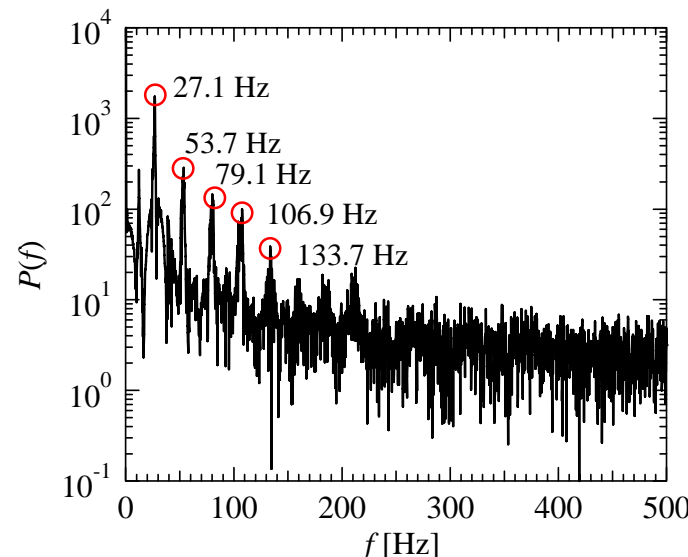

Fig. 7: FFT analysis of the aspect ratio of the oscillating droplet

Table 2: Comparison between experimental result and theory

\begin{tabular}{cccccc}
\hline$n$ & 2 & 3 & 4 & 5 & 6 \\
\hline Theory [Hz] & 28.7 & 55.6 & 86.1 & 120.1 & 157.2 \\
\hline Experiment [Hz] & 27.1 & 53.7 & 79.1 & 106.9 & 133.7 \\
\hline Disagreement [\%] & 5.6 & 2.9 & 8.1 & 11 & 14.9 \\
\hline
\end{tabular}

In table 2, there is the comparison between result of FFT analysis and resonance frequencies estimated by equation (5). We defined resonance frequencies of the experiment as each peaks of power spectrum density. The experimental result is good agreement with theoretical approach. We confirmed that the shape oscillation shown from the experiment is dominated by the combination from 2 to 6 order mode of deformation. The frequencies, which $n$ is over 5 have disagreement of more than $10 \%$. This is because nonlinear effect due to the different condition between experiment and theory. Though the theory assumed an inviscid droplet and small oscillation, the real droplet has viscosity and oscillates largely. According to this result, it is indicated that a heated droplet oscillates with its resonance frequencies.

\section{Simulations of an Oscillating Droplet}

We reconstruct a motion of the droplet analytically. The radius of spherical surface at an arbitrary moment $r$ is expressed below:

$r(\theta, t)=R_{0}+\sum_{n=2}^{\infty} A_{n} \cos \left(2 \pi f_{n} t\right) P_{n}^{m}(\cos \theta)$,

where $R_{0}$ is the initial radius of sphere, the $A_{n}$ is amplitude of $n$-order mode, the $f_{n}$ is frequency of $n$-order mode, $\theta$ is the angle, $t$ is the time and $P_{n}{ }^{m}$ is Legendre function. The values of resonance frequency $f_{\mathrm{n}}$ and power spectrum density $P\left(f_{\mathrm{n}}\right)$ from FFT analysis in Fig. 7 is applied to this equation (6). The $m$ of $P_{n}{ }^{m}$ is assumed as 0 because the horizontal shape oscillation was experimentally observed. The $A_{n}$ is derived from $P\left(f_{n}\right)$ as follows:

$A_{n}=\frac{1}{2} \sqrt{\frac{P\left(f_{n}\right)}{f_{n}}}$.

Figure 8 shows one cycle of the shape oscillation reconstructed from the combination of experimental data and equation (6). The ultrasonically levitated droplets were more flattened than the simulated droplet because the equation (6) is not considered the acoustic force and gravity. It is indicated that there is such nonlinear effect due to the difference between equation (6) and experiment. However, the simulation qualitatively makes agreement with experimental result. It is clarified that the surface deformation can be reconstructed by using the equation (5), (6), (7) and the experimental data from 2 to 6 order modes which includes power spectrum densities and the resonance frequencies.

In order to investigate the effect of high order modes on the oscillation, we only applied the combination of $f_{2}$ and $P\left(f_{2}\right)$ from Fig. 7 to the equations of (6) and (7). Fig. 9 shows the shape oscillation of the droplet simulated by using the values of 2-order mode. There are apparent difference between Fig. 8 and Fig. 9. The simulation of Fig.8 has 
complex deformations due to high order modes over $n=3$. It is clarified that the oscillation of a heated droplet includes high order modes and the modes affect on the shape of oscillations.

Finally, we examined the influence of droplet temperature on the shape of the deformation. We changed the values of $\sigma_{\mathrm{s}}$ as $62.6 \mathrm{mN} / \mathrm{m}$ and $\rho_{\mathrm{s}}$ as 972 $\mathrm{kg} / \mathrm{m}^{3}$. The $\sigma_{\mathrm{s}}$ and the $\rho_{\mathrm{s}}$ were assumed that the water droplet is $80^{\circ} \mathrm{C}$. Figure 10 shows the simulation of the shape oscillation on the condition that the $\sigma_{\mathrm{s}}$ is $62.6 \mathrm{mN} / \mathrm{m}$ and $\rho_{\mathrm{s}}$ is $972 \mathrm{~kg} / \mathrm{m}^{3}$. The other values such as amplitude of each mode of oscillations except for resonance frequencies are same as the Fig. 8. The resonance frequencies obtained from equation (5) were changed due to variation of $\sigma_{\mathrm{s}}$ and $\rho_{\mathrm{s}}$. Compared to the Fig. 8, the shapes of Fig. 10 are almost same mainly because the power spectrum densities were used from the Fig. 7, which is not supposed to be the same. Therefore, in order to improve the accuracy of the simulation, the further investigation is needed.
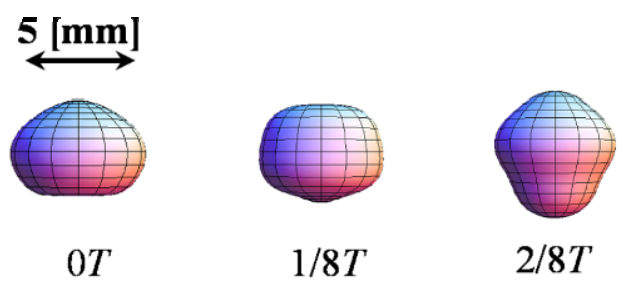

$2 / 8 T$
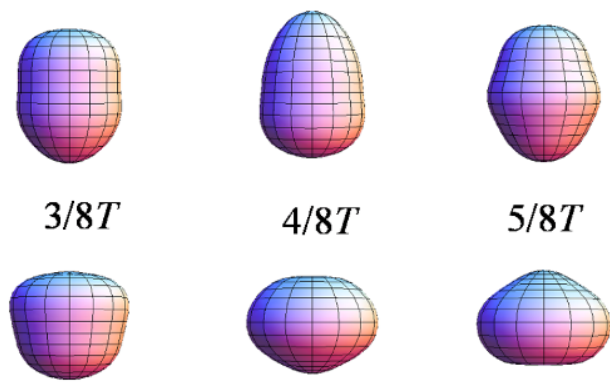

$6 / 8 T$

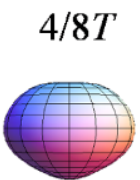

$7 / 8 T$

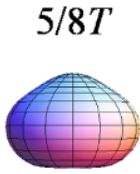

$1 T$

Fig. 8: Simulation of one cycle of oscillation using the values of 2 to 6 order modes

$\left(\sigma_{\mathrm{s}}=70.4 \mathrm{mN} / \mathrm{m}\right.$ and $\rho_{\mathrm{s}}=994 \mathrm{~kg} / \mathrm{m}^{3}$ for $T_{\mathrm{s}}=$ $\left.35^{\circ} \mathrm{C}\right)$

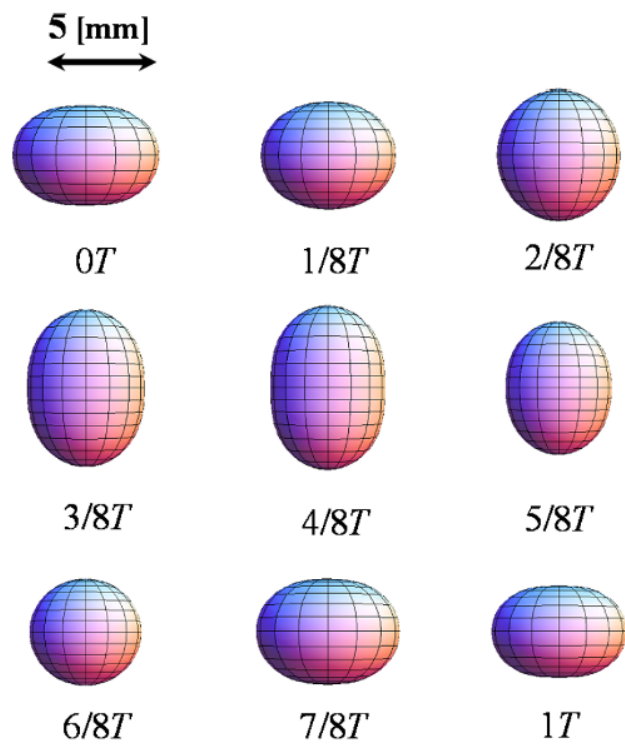

Fig. 9: Simulation of one cycle of oscillation using the values of 2-order mode

$\left(\sigma_{\mathrm{s}}=70.4 \mathrm{mN} / \mathrm{m}\right.$ and $\rho_{\mathrm{s}}=994 \mathrm{~kg} / \mathrm{m}^{3}$ for $T_{\mathrm{s}}=$ $\left.35^{\circ} \mathrm{C}\right)$
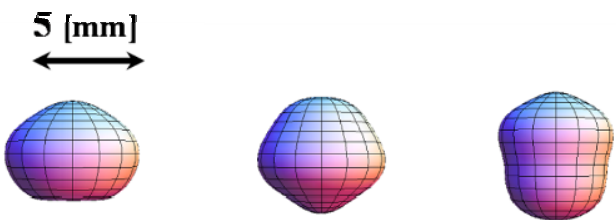

$0 T$

$1 / 8 T$

$2 / 8 T$
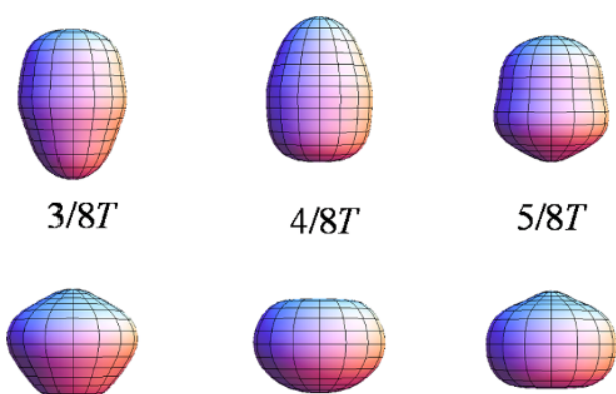

$5 / 8 T$
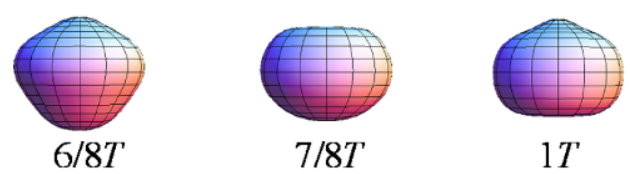

$1 T$

Fig. 10: Simulation of one cycle of oscillation using the values of 2 to 6 order modes

$\left(\sigma_{\mathrm{s}}=62.6 \mathrm{mN} / \mathrm{m}\right.$ and $\rho_{\mathrm{s}}=972 \mathrm{~kg} / \mathrm{m}^{3}$ for $T_{\mathrm{s}}=$ $\left.80^{\circ} \mathrm{C}\right)$ 


\section{Conclusions}

In the present study, shape oscillation of a heated droplet was experimentally obtained and simulated by using the experimental data of FFT analysis. Following discussions were acquired.

(1) A periodical deformation of the droplet was observed when the droplet was heated. Compared the theory of equation (5) with FFT analysis of the experimental result, there was good agreement. It is confirmed that the heated droplet oscillated with resonance frequencies and that the oscillation included 2 to 6 order modes.

(2) The shape oscillation of the heated droplet was reconstructed from our experimental data. We can find that the shapes of simulated oscillation were not completely same as snapshots obtained from experiment due to the differences between assumption of the theory and experimental conditions.

(3) Summing up the conclusions of (1) and (2), it is indicated that the periodical deformation of heated droplet is composed of resonance frequencies and nonlinear oscillation.

\section{References}

Abe, Y. et al., Interfacial Stability and Internal Flow of a Levitated Droplet. Microgravity Sci. Technol. Vol. 19(3-4), 33-34 (2007)

Danilov, S. D. et al., Breakup of a droplet in a high-intensity sound field, J. Acoust. Soc. Am. 92(5), 2747-2755

Ermoline, A. et al., Experimental technique for studying high-temperature phase in reactive molten metal based systems, Rev. Sci. Instrum. Vol. 76, 5177-5185 (2004)

Kawakami, M. et al., Effect of Temperature Change on Interfacial Behavior of an Acoustically Levitated Droplet, Microgravity Sci. Technol

King, L. V., On the acoustic Radiation Pressure on Spheres. Proc. R. Soc London, Ser. A 147, 212 (1934)

Marston, P. L., Shape oscillation and static deformation of drops and bubbles driven by modulated radiation stresses - theory.

J. Acoust. Soc. Am. 67(1), 15-26 (1980)

Marston, P. L., Quadrupole projection of the radiation pressure on a compressible sphere. J. Acoust. Soc. Am. 69(5), 1499-1501 (1981) Rayleigh, L., On the Capillary Phenomena of Jets. Proc. Roy. Soc. Vol. 29, 71-97 (1879) Trinh, E.H. et al., An Experimental study of small-amplitude drop oscillations in immiscible liquid systems. J. Fluid Mech.

Vol. 115, 453-474 (1982)

Wang, T. G. et al., Oscillations of liquid drops: results from USML-1 experiments in Space. J. Fluid Mech. Vol. 308, 1-14 (1996)

Xie, W. J. et al., Eutectic growth under acoustic levitation conditions.

Phys. Rev. E 66, 061601 (2002) 